Sufficient studies for meta-analytic review were in three domains: knowledge, attitudes, and sexual behaviours. To examine main intervention effects within each domain, study's intervention characteristics were extracted to compute the pooled Hedges' $g$ and $95 \%$ CIs using the random-effects model. Effect size heterogeneity was measured by $\mathrm{I}^{2}$.

Results From 9064 candidates, 21 studies across three domains were included in the meta-analysis. For knowledge, there was a large intervention effect on sexual health knowledge, Hedges' $g=1.32(0.51-2.14)$. The pooled effect size for both attitudes and sexual behaviour domains yielded small to medium intervention effects, Hedges' g $=0.58(0.34-0.81)$ and 0.25 (0.13-0.37), respectively. Heterogeneity for each domain was substantial (between $91 \%$ and $97 \%$ ). Removal of outliers decreased heterogeneity estimates for all domains ( $21 \%$ to $62 \%$ ), i.e., small. The final effects sizes for knowledge, attitudes and sexual behaviour were 0.25 $(0.03-0.48), 0.21(0.15-0.28)$ and $0.10(0.06-0.15)$, respectively.

Conclusions Our results indicate that existing intervention efforts focused on sexual health knowledge, attitudes and behaviour have small, but positive significant effects. Studies were not designed to encompass all the domains of sexual health, but when studies were examined by these domains the results were consistent across the studies.

\section{P4.113 STIGMA, DISCRIMINATION AND UNDUE PRESSURE FACED BY MSM'S FROM THE FAMILY, PEERS AND SOCIETY LEADING TO A DOUBLE STANDARD LIFE}

doi:10.1136/sextrans-2013-051184.1010

'Z Haq, 'R Akber, ${ }^{2}$ Oasim, ${ }^{2} \mathrm{R}$ Bushra, ${ }^{3} \mathrm{~K}$ Sheryar. ${ }^{1}$ parwaz male health society, karachi, Pakistan, ${ }^{2}$ Naz Male Health Alliance, Lahore, Pakistan, ${ }^{3}$ Naz Male Health Alliance, karachi, Pakistan

Background Parwaz Male Health Society is implementing GFATM's Regional Grant R-9 to empower MSM/TG communities in Pakistan to prevent HIV/AIDS, under the technical assistance provided by Naz Male Health Alliance, Pakistan. Participatory research methods is used to reach MSM and understand community needs to effectively design prevention-programmes in Karachi, where MSM's are engaged in high risk behaviours to an alarmingly high level which makes them vulnerable to HIV/AIDS.

Methods A case study approach was used to analyse data involving the MSM community members; Malishias, truck/cab/drivers, migrant, labour, and self identified gay people etc. visiting the Drop in Center. 12 cases were studied and Data was collected through In-depth interviews, participant's observation and data verification, done through the field workers, Community counsellor and the Doctor. Ethical standards mentioned in NMHA's Institutional manual.

Results Ranging between the age group 16-50 years, MSM's have been subjected to labelizing, stigmatisation, discrimination since childhood within home and the external world, whether educated or uneducated, financially stable or not, low self esteem, lack of understanding and knowledge on self identity and human rights, facing violence/bullying from around, forced decisions about marriage, career and religious practises, altered mental and physical development and a damaged personality, hampering their productivity and efficiency to contribute to society in a healthy way.

Conclusion An urgent need for an all age group focused programming covering Psycho social and sexual health, strategies to cope with bullying and pressure inflicted including counselling and self help group therapy, acceptance of self identity, socio-economic empowerment, education and awareness along HIV and Sexually Transmitted Infections prevention. Further qualitative and quantitative evidence is required to effectively design programmes.

\section{P4.114 SEXUAL BEHAVIOR AND PRACTICE AMONG TRANSGENDERS IN WEST BENGAL, INDIA}

doi:10.1136/sextrans-2013-051184.1011

M Kundu, N Panda, S Chowdhury, S Das konar, R Basu, D Das, R Sonkar, A RoyChoudhury. Child In Need Institute, Kolkata, India

HIV Epidemic in India is not generalised but is a concentrated epidemic. The Prevalence of HIV among Transgender is 8.82, thus there is the necessity for specific programmes. Hence, a study among the transgender conducted to know the sexual behaviour \& practise among them.

A structured questionnaire had been developed followed by training of the interviewer. The respond Driven Sampling method was used. The study had been conducted in collaboration with Community Based Organization working with Transgender in West Bengal.

According to this study the $30 \% \& 42 \%$ respondents have secondary and graduate level of education whereas $28 \%$ are post graduate. $25 \%$ populations below 18 years have more than 10 sexual partner and 18 years above have 7 partners on an average in a month. $43 \%$ respondents have exposure of sexual intercourse before the age of 13 \& rests $57 \%$ between the ages 14 to $18.12 \%$ are married with female but all of them preferred \& practise anal sex and have multiple male sexual partners. $81 \%$ contact with their partners through mobile phone or use e-communication which hampers the so called Outreach concept. $88 \%$ do not know about the sign \& symptoms of STI but $67 \%$ of them being affected with it. $83 \%$ are inconsistent condom user and they have complained of condom rupture during sex \& unavailability of lubricant. $80 \%$ populations have self risk perception to get HIV.

Indian National HIV Programme Design need to cater specific need based tailor made Transgender Programme. Need convergence of Adolescent \& HIV Programme for Transgender. Incorporate Social Media, phone in new outreach strategy for more reaching out. National HIV prevention Programme needs specific male STI Guideline. The Self Risk perception among this community is very high so immediate new programme strategy will help the Transgender Community to prevent HIV.

\section{P4.115 THE FORGOTTEN STI: A SURVEY OF KNOWLEDGE ABOUT TRICHOMONIASIS}

doi:10.1136/sextrans-2013-051184.1012

'D Arrindell, 'L Barclay, ${ }^{2} \mathrm{~J}$ Boxt. 'American Sexual Health Association, Durham, NC, United States, ${ }^{2}$ Glover Park Group, Washington, DC, United States

Background Trichomoniasis is the most common curable STI in the US. According to the Centers for Disease Control and Prevention, an estimated 7.4 million new cases occur each year. Trich is a largely hidden STI - out of the public eye, and absent from serious discussions about STI prevention. Increasing awareness requires improved understanding of what women know to develop messages to encourage testing.

Methods A survey was conducted on behalf of American Sexual Health Association via an online panel by Research Now, an independent research company. Interviews were conducted between January $28^{\text {th }}$ - February $2^{\text {nd }}, 2013$ among a nationally representative sample of 1,000 female respondents between the ages of 18 and 50 .

Results Women surveyed perceive trich as the least common STI; only one in five $(22 \%)$ women are familiar with it. Sixty-five percent would not seek medical attention if they experienced unusual symptoms, instead waiting to see if the symptoms go away or treating themselves with over-the-counter medicine. Those most likely to self-treat include Hispanic women (33\%), those with a high school or less education (33\%), and Southerners (32\%). Among 